\title{
Equipe de apoio e a construção coletiva do trabalho em Saúde Mental junto à Estratégia de Saúde da Família: espaço de discussão e de cuidado
}

Support team and collective elaboration of the Mental Healthcare work within the Family Health Program: space of discussion and care

\author{
Maria Margarida Licursi Prates \\ Especialista em Psicanálise e em Saúde Mental. Terapeuta Ocu- \\ pacional da Unidade Básica de Saúde Vila Romana da Secretaria \\ Municipal da Saúde de São Paulo (aposentada). Pesquisadora \\ do Laboratório de Saúde Mental Coletiva da Faculdade de Saúde \\ Pública da USP. \\ Endereço: Rua Iperoig, 561, ap. 114, Perdizes, CEP 05016-000, São \\ Paulo, SP, Brasil. \\ E-mail: mlicursipratesळyahoo.com.br

\section{Vânia Ghirello Garcia} \\ Especialista em Psicanálise e em Saúde Pública. Psicóloga da \\ Unidade Básica de Saúde Vila Romana da Secretaria Municipal \\ da Saúde de São Paulo. \\ Endereço: Rua Iperoig, 554, ap. 102, Perdizes, CEP 05016-000, São \\ Paulo, SP, Brasil. \\ E-mail: vaniagg®terra.com.br

\section{Diva Maria Faleiros Camargo Moreno} \\ Psicóloga. Mestre e Doutora em Saúde Pública. Coordenadora da \\ Unidade Básica de Saúde Vila Anastácio da Secretaria Municipal \\ da Saúde de São Paulo. Pesquisadora do Laboratório de Saúde \\ Mental Coletiva da Faculdade de Saúde Pública da USP. \\ Endereço: Rua Iperoig, 690, ap. 173, Perdizes, CEP 05016-000, São \\ Paulo, SP, Brasil. \\ E-mail: dmfcmळusp.br
}

\section{Resumo}

A grande demanda por consulta psiquiátrica e psicoterapia e a necessidade de otimizar os recursos humanos em Saúde Mental têm conduzido à organização de novos modos de ação e suporte técnico aos profissionais de unidades com Estratégia de Saúde da Família. Uma Unidade Básica de Saúde de uma região da cidade de São Paulo iniciou a prática denominada Apoio Matricial, que consiste no suporte à identificação, tratamento, e proposta de solução dos problemas de saúde mental em conjunto com as equipes de Saúde da Família. O presente trabalho tem por objetivo relatar a experiência focada nesse processo de trabalho, tendo como horizonte ético os princípios do Sistema Único de Saúde e concepções técnicas e científicas mais humanizadas com vistas ao atendimento integral ao paciente e a sua inserção no meio social. Trata-se de um estudo de natureza qualitativa que utilizou a observação participante como meio para coletar os dados e analisar a situação. Consoante com a Reforma Psiquiátrica no Brasil, busca-se uma visão complementar e menos fragmentada do atendimento em Saúde Mental. A experiência mostrou que por meio do apoio matricial pode ser alcançada a necessária (re)organização dos serviços para acolher a demanda e efetivar a atenção em Saúde Mental de qualidade. Esse modo de operar, ao integrar Equipes de Saúde da Família e Unidade Básica Tradicional, permitiu a construção compartilhada do cuidado em saúde.

Palavras-chave: Saúde Mental; Serviços de Saúde Mental; Apoio Matricial; Rede de Saúde. 


\section{Abstract}

The great demand for psychiatric and psychotherapeutic care and the need to optimise human resources in Mental Health have led to the development of new practices of care and technical support to professionals of health services with Family Health Program. A Primary Care Service in the city of São Paulo introduced Matrix Support, a practice that provides help in identifying, caring for and proposing new solutions for the mental health problems together with the Family Health workers. This study aims to report the experience of this work process, having the principles of the Health System and techno-scientific concepts more humanized as ethic north, directed not only to the patient's integral care but also their social insertion. It is a qualitative study that used the participant observation to collect data and analyse the situation. According to the Brazilian Psychiatric Reform, this initiative searches for a complementary and integral vision of Mental Healthcare. The experience showed that matrix support is effective in reaching the necessary (re)organization of health services in order to receive people and offer a Mental Health care of quality. This practice of integrated work between Family Health and Traditional Primary Care teams contributed to a shared construction of healthcare. Keywords: Mental Health; Mental Health Services; Matrix Support; Health's Network.

\section{Introdução}

A Saúde Mental Pública no Brasil se modificou e vem sendo construída gradativamente para atender aos anseios dos profissionais, usuários do sistema e seus familiares. Entre os anos 1980 e 1990, o movimento da Reforma Psiquiátrica e o da Reforma Sanitária tomam força pelos eventos históricos e políticos representados pela $8^{\mathrm{a}}$ Conferência Nacional de Saúde, em 1986, e pela $1^{\underline{a}}$ Conferência Nacional de Saúde Mental, em 1987 (Hirdes, 2009). A proposta de reorganização da assistência psiquiátrica é sustentada e encampada pelo Brasil com a elaboração do Sistema Único de Saúde (SUS) a partir da Constituição de 1988. Essa proposta visa oferecer aos portadores de transtornos mentais uma nova lógica de atenção à saúde e a transformação das condições de exclusão social nas quais eles se encontram em decorrência de suas patologias e formas de tratamento manicomiais vigentes na sociedade até aquele momento.

Tendo em vista as diretrizes estabelecidas pelo SUS, a legislação brasileira redireciona o modelo assistencial, em respeito aos direitos das pessoas portadoras de transtornos mentais (Brasil, 2001). A pessoa portadora de transtornos mentais deve ter acesso ao melhor tratamento do sistema de saúde, sendo cuidada com humanidade e respeito para beneficiar sua saúde e alcançar sua recuperação por meio da inserção na família, no trabalho e na comunidade. Ela deve ser protegida contra qualquer forma de abuso e exploração, com garantia de sigilo no atendimento. Deverá também ter a presença médica para esclarecer a necessidade ou não de sua hospitalização involuntária, recebendo o máximo de informações a respeito de sua doença e de seu tratamento, o ambiente terapêutico sendo o menos invasivo possível. O tratamento em serviços comunitários de saúde mental, como os Centros de Atenção Psicossocial (CAPS), é o preferível (Brasil, 2005, p. 49).

Na atual política de saúde, os CAPS são dispositivos estratégicos para a organização da rede de atenção em saúde mental (Brasil, 2004). Porém, CAPS não é o único tipo de serviço que é oferecido à população, uma vez que a atenção em saúde mental deve ser feita dentro de uma rede de cuidados incluindo vários níveis de serviços, tais como: unidades básicas de saúde, ambulatórios, centros 
de convivência, residências terapêuticas, clubes de lazer, entre outros (Brasil, 2004).

No SUS a porta de entrada deve ser a Unidade Básica de Saúde (UBS), quer as tradicionais quer aquelas com Estratégia de Saúde da Família (ESF). No entanto, em se tratando de Saúde Mental, o CAPS tem a missão de regulador do fluxo dos atendimentos dentro de sua área de abrangência. Agrega-se a isso sua função primordial da assistência aos problemas mais complexos de saúde mental (Brasil, 2004). Como elemento intermediário entre os equipamentos primários (UBS) e secundários (CAPS), encontram-se as equipes de apoio, ou de referência, hoje os Núcleos de Apoio à Saúde da Família (NASF), para instrumentalizar especificamente as equipes de Saúde da Família na tarefa de dar conta, entre outros, dos problemas de saúde mental de sua região (Brasil, 2008b).

O modelo implantado e que vem sendo reforçado pelo governo que incentivou a criação dos CAPSs e dos NASFs é o da Reabilitação Psicossocial, considerado como o paradigma atual da Atenção em Saúde Mental.

O termo psicossocial designa práticas em Saúde Mental Coletiva com a função de substituir o modelo assistencial psiquiátrico ainda dominante (Costa-Rosa e col., 2003). O uso desse termo visa incluir nas práticas aspectos políticos, econômicos, valores culturais como componentes de um sentido mais amplo de articulação.

Costa-Rosa (200o) analisa quatro parâmetros que definem o "modo psicossocial", expressão utilizada por esse autor: a) concepções do processo saúde-doença e dos meios teórico-técnicos para lidar com ela; b) concepções da organização das relações institucionais e do trabalho interdisciplinar; c) concepção da forma de relações da instituição e de seus agentes com a população e d) concepções dos efeitos das ações em termos terapêuticos e éticos. 0 que baliza estes aspectos é a noção de humanização, compreendida como a valorização dos diferentes sujeitos implicados no processo de produção de saúde. Com base nessa descrição, pode-se entender a atenção psicossocial como referencial ético-teórico-político do movimento da Reforma Psiquiátrica no Brasil e como modo prático de cuidado em saúde mental (Costa-Rosa, 200o), sempre se levando em conta o contexto social e institucional. Assim, o trabalho em saúde mental é norteado por valores como a autonomia e o protagonismo dos sujeitos, além da corresponsabilidade entre eles, os vínculos solidários e a participação coletiva no processo de gestão (Brasil, 2006; Campos e col., 2009).

Neste contexto, o modelo assistencial conhecido como "Apoio Matricial e Equipe de referência" (Moysés, 1992; Campos, 1999; Brasil, 2004; Campos e Domitti, 2007) nos serve de base para a discussão da experiência, pois pressupõe o atendimento humanizado, participativo e com corresponsabilização entre os diversos atores sociais.

A concepção matricial pressupõe uma organização de trabalho que valoriza a integração e a descentralização, isto é: há uma oposição à departamentalização e à segmentação em especialidades, ao mesmo tempo em que favorece a autonomização (Moysés, 1992).

Considerado como uma metodologia de trabalho, o apoio matricial visa assegurar retaguarda especializada a equipes e profissionais encarregados da atenção a problemas de saúde, incluída a saúde mental (Campos e Domitti, 2007). Em sua forma de operar está previsto, além da assistência, o suporte técnico-pedagógico necessário ao desenvolvimento do conhecimento e troca de saberes que possibilitem a construção compartilhada de responsabilização.

Nesse sentido, ampliar a discussão das questões de saúde mental entre os profissionais especializados com os da atenção básica traz a vantagem de um trânsito maior do sujeito nessa instituição de saúde, aliado à responsabilidade das Unidades Básicas com Estratégias de Saúde da Família (UBSFs) em relação ao usuário. Isso se torna possível uma vez que entre as características desse nível da atenção à saúde estão a integralidade, a continuidade ou longitudinalidade e a coordenação do cuidado (Starfield, 2002).

A equipe ou profissional de referência tem ao seu encargo a condução do caso, seja um indivíduo, uma família, seja uma comunidade. 0 apoiador matricial é o especialista em saúde mental (um profissional ou uma equipe interdisciplinar) a oferecer retaguarda. O arranjo tem como objetivo o desenvolvimento da clínica ampliada e a integração dialógica entre as especialidades das equipes de referência e de apoio (Campos e Domitti, 2007). 
A clínica ampliada é uma concepção epistemológica e organizacional que se baseia na construção do trabalho clínico segundo certo traço artesanal por meio do qual a divisão do processo de trabalho é acompanhada da responsabilização dos profissionais de um modo mais integrado. 0 objeto de trabalho é ampliado para além das doenças, incluindo também questões de saúde, que são as situações envolvendo a vida e a vulnerabilidade das pessoas (Campos e Amaral, 2007). "A ampliação mais importante, contudo, seria a consideração de que, em concreto, não há problema de saúde ou doença sem que estejam encarnadas em sujeitos, em pessoas." (Campos e Amaral, 2007, p. 852).

Esta proposta de trabalho tem a finalidade de promover a autonomia dos sujeitos envolvidos (profissionais e usuários) e o momento da implantação da presente experiência foi de grande auxílio no sentido de prover os profissionais de uma tecnologia de trabalho em Saúde Mental rica em possibilidades de ação e cuidado.

Tendo em vista essas concepções e o momento em que se encontra a política pública do SUS, o presente estudo tem por objetivo descrever a experiência de implantação do Apoio Matricial em Saúde Mental na Atenção Básica, focada no processo de trabalho de uma equipe de saúde mental junto à Estratégia de Saúde da Família.

\section{Como e de onde partiu a construção da experiência}

A experiência aqui relatada originou-se de uma solicitação institucional para a reorganização do acesso ao cuidado em saúde mental na Atenção Básica, a partir da necessidade de otimização dos recursos humanos especializados. Os profissionais com essa capacitação foram estrategicamente concentrados em algumas unidades de saúde, denominadas de “Apoio em Saúde Mental”, que serviriam de referência para determinadas Unidades Básicas com Estratégia de Saúde da Família (UBSF).

Na presente experiência foram envolvidas duas UBSFs que se localizavam na periferia de uma região da cidade de São Paulo, sendo a unidade de apoio - mais central - pertencente à mesma região administrativa de saúde. A proposta consistiu em oferecer suporte técnico e organizar a cobertura do atendimento à população dessas UBSFs pela equipe de saúde mental ("Equipe de Apoio"), composta por: duas psicólogas, duas terapeutas ocupacionais e uma psiquiatra, sendo duas delas, autoras do presente artigo.

As UBSFs estavam estruturadas em seis equipes compostas por: um médico generalista, um enfermeiro, dois auxiliares de enfermagem e seis ou sete agentes comunitários de saúde (ACS). Nem sempre essas equipes estavam completas, devido a demissões, licenças ou outros motivos.

Cada equipe é uma verdadeira célula da estrutura da ESF, tendo sob sua responsabilidade cerca de mil famílias, ou seja, em torno de cinco mil pessoas.

O diferencial desse programa consiste na abordagem domiciliar por esses profissionais a famílias moradoras em determinado território e no seguimento destas em suas necessidades de saúde visando à promoção, à prevenção e à solução dos problemas de saúde compatíveis com o nível da atenção primária. Esta abordagem domiciliar, bem como o conhecimento e apropriação do território foi bastante valorizado pela equipe de apoio em seu trabalho, pois é essencial na Estratégia de Saúde da Família.

A concepção de território compreende não só o espaço físico, mas todo o ambiente social e cultural do indivíduo. Ou seja: abre-se a possibilidade de pensar no lugar onde as pessoas vivem de modo efetivo ou potencialmente, e a sua importância “...decorre de suas próprias virtualidades, naturais ou sociais, preexistentes ou adquiridas segundo intervenções seletivas. (Santos, 1988, p. 11) Caracteriza-se assim, a apropriação de todos os recursos que se mostram acessíveis para suprir as necessidades dos sujeitos ali inseridos. São aspectos importantes a serem considerados para a abordagem das questões suscitadas pelo modelo de atenção proposto, não só relativas à saúde mental, mas a todo o sistema de saúde.

Nessa concepção adotada, as ações são focadas nas necessidades dos sujeitos em seu território e as formas de produzir saúde passam pela modificação do processo de trabalho das equipes (Barros e col., 2007).

O território onde as duas UBSFs estavam inseridas agregava: escolas de educação infantil, de ensino fundamental e médio, igrejas com movimento 
pastoral e promoção de grupos de artesanato que ocorriam em um espaço comunitário. Além disso, havia a participação efetiva dos membros da comunidade nos conselhos de saúde e nas ações de promoção da saúde nas unidades e em seu entorno. Havia festas periódicas nas praças próximas às UBSFs, com o envolvimento dos agentes de saúde e de outros profissionais.

Todas as ações da equipe de apoio buscavam favorecer a acessibilidade aos serviços, a integralidade em saúde e a equidade, princípios básicos do SUS. E, para que houvesse resolubilidade, contava-se com a rede de atenção em saúde, com seus serviços de média e alta complexidade tecnológica - UBSs tradicionais, CAPS, ambulatórios de especialidades e hospitais -, atendendo ao princípio de hierarquização do SUS. Nessa região, as UBSFs estavam integradas aos seguintes serviços de saúde mental: um Pronto-Socorro, com psiquiatra e leitos para 72 horas; um Ambulatório de Especialidades; três Centros de Atenção Psicossocial, sendo um Adulto, um Infantil e um Álcool e Drogas; um Centro de Convivência e Cooperativa (CECCO) e a UBS de Apoio em Saúde Mental da qual o presente trabalho se originou.

\section{A experiência em si}

Este trabalho, anterior à implantação dos NASFs, compreendeu o período de 2005 a 2008.

A intervenção consistiu na construção de um processo de trabalho compartilhado entre a equipe de apoio e as equipes da ESF para: a) a identificação dos problemas de saúde mental da população adscrita b) o tratamento dos problemas de saúde mental de menor complexidade, propondo soluções e seguindo os casos em conjunto com todos os integrantes da equipe e c) encaminhamento dos casos mais graves ao CAPS ou outro serviço de referência quando necessitassem de tratamento especializado. Tal estratégia oferecia um novo olhar sobre o cuidado aos portadores de transtornos mentais e a interação com os profissionais da equipe de referência na medida em que tinha como ideia conceitual de base a atenção psicossocial, contrariamente ao modelo manicomial, em que os usuários são encaminhados para tratamento e/ou internação, especialmente centrado no profissional médico não se valorizando seus recursos mais saudáveis, nem seu contexto de vida e comunidade, ou os saberes dos outros profissionais das equipes.

O processo de trabalho teve início com a formação de duplas de profissionais da unidade de apoio que se deslocavam com veículo institucional até a UBSF para acompanhar de perto o trabalho das equipes, de forma a dar-lhes suporte técnico-pedagógico. Isso ocorria nos períodos da manhã e da tarde por duplas diferentes de profissionais. Foi estabelecido um cronograma de visitas semanais às UBSFs, de modo a permitir que cada equipe pudesse se reunir com os profissionais do apoio uma vez por mês. A dinâmica das reuniões baseava-se na discussão dos casos eleitos pela ESF, com a participação de agentes comunitários de saúde, auxiliares de enfermagem, enfermeiro e médico. 0 critério de encerramento da discussão era a compreensão do problema e a elaboração conjunta de proposta de ações a serem desenvolvidas para dar resposta à demanda (do usuário ou da própria equipe). Frequentemente, neste espaço de discussão era delineado o projeto terapêutico para cada caso.

Tanto na elaboração do diagnóstico quanto do projeto terapêutico, as observações e impressões de todos os participantes eram levadas em consideração, bem como o potencial de cada um em poder realizar as tarefas necessárias para o melhor desfecho do caso. Neste sentido, a contribuição dos ACS com elementos necessários à compreensão dos casos era valiosa, pois eles dispunham de informações coletadas em suas visitas domiciliares e em sua interação com os usuários, em conversas formais e informais no cotidiano do território. O ACS é a pessoa que convive com a realidade e as práticas de saúde do bairro, pois nele mora e trabalha munido de conhecimentos em torno do processo de saúde-doença, e de saberes que lhe servem como ferramenta na interação cotidiana com as famílias e no reconhecimento de suas necessidades (Nunes e col., 2002).

As informações relativas às discussões de cada reunião eram registradas de modo sistemático em um fichário mantido na unidade de apoio, uma espécie de diário de campo, permitindo a sistematização dos dados e a presente reflexão e relato.

A Unidade de Apoio criou um modo de recepção, no qual os casos indicados para a psiquiatra eram 
acolhidos por uma dupla de profissionais que realizavam uma escuta qualificada para responder à necessidade da demanda, havendo registro dos atendimentos em prontuários específicos para os casos encaminhados pelas equipes de referência da ESF. Desse modo o Acolhimento funcionava como instrumento de gestão dos casos e da própria operacionalidade do modelo assistencial proposto. Em geral, a dupla era constituída pela psiquiatra e a terapeuta ocupacional.

Outra providência foi organizar dispositivos de atendimento compartilhado dos casos como grupos de acolhimento, grupos terapêuticos diversos realizados por psicólogos, terapeutas ocupacionais, e pela psiquiatra. Às vezes, quando indicado, o atendimento individual era providenciado na UBS de Apoio.

\section{Reflexões metodológicas}

0 presente estudo é de natureza qualitativa, estando circunscrito a uma situação local e temporal. Procedeu-se a uma observação sistemática e participante de todos os encontros ao longo do período de realização, com registros por mais de um observador em cada uma das etapas do processo de trabalho: escolha dos casos, sua discussão técnica e fenomenológica, elaboração do projeto terapêutico de cada caso, encaminhamentos necessários e acompanhamento da evolução terapêutica. Neste tipo de enfoque, o observador torna-se parte integrante do processo de conhecimento e descoberta, fazendo uma seleção do que será interpretado no relato (Peirano, 1995). É uma estratégia que combina simultaneamente a análise de documentos, a entrevista de respondente e informante, a participação e observação direta e a introspecção (Denzin, 1989 apud Flick, 2009, p. 207).

Como o observador está presente no campo, as observações contêm a perspectiva de elemento das situações e ambientes específicos envolvidos, com interesse especial no pensamento e na interação humana. Há uma ênfase na interpretação e compreensão da existência humana, com redefinição constante do que é considerado problemático e relacionado ao objeto de estudo (Flick, 2009). A observação permite absorver em profundidade as relações entre os participantes por meio dos proces- sos comunicacionais estabelecidos.

Como já mencionado, os dados foram coletados a partir dos registros em diário de campo e em prontuários dos atendidos na unidade de apoio. A análise enfocou os objetivos da implantação do processo de trabalho matricial propriamente dito, quais sejam: a necessidade de mudança de paradigma no atendimento em saúde mental, a instrumentalização técnica dos profissionais da UBSF para o trabalho em Saúde Mental, acolhimento dos usuários na unidade de apoio, a otimização dos recursos em saúde mental.

\section{Resultados e discussão}

No momento da implantação do trabalho, alguns problemas começaram a ser identificados e podem ser mencionados: a) demanda excessiva; b) prevalência do modelo assistencial psiquiátrico para tratamento da maioria dos usuários, em detrimento de abordagens complementares e substitutivas; e c) despreparo dos profissionais da ESF para abordar os problemas de saúde mental.

O enfrentamento desses problemas partiu da reflexão conjunta sobre os modos de conceber e de lidar com a doença e com o doente mental. Discutia-se continuamente a possibilidade de incluir novas abordagens e tecnologias nas práticas dos profissionais, como por exemplo, uma visita domiciliar realizada pela enfermeira objetivando a orientação ao paciente ou à família em relação a determinados aspectos salientados na discussão do caso. Com esse tipo de proposição, buscou-se valorizar e viabilizar a exploração de recursos das equipes e do território, otimizando os processos de trabalho em substituição a soluções imediatas e repetitivas para os problemas de saúde mental trazidos para as reuniões.

As proposições de respostas alternativas para abordagem dos problemas de saúde mental, a princípio, eram vistas com descrédito, diante da expectativa de obter o tratamento convencional, baseado em uma racionalidade tecnológica já conhecida e utilizada no serviço, como o encaminhamento direto da consulta médica para o especialista em doenças mentais. Isso era ocasionalmente mencionado por alguns usuários e por alguns profissionais, de ambas as equipes (da ESF e do apoio), enfatizando uma lógi- 
ca de medicalização e tratamento médico centrado.

Nesses casos notavam-se algumas resistências em aceitar as condutas propostas tais como atendimentos em grupo, avaliação em dupla de profissionais, terapias alternativas como caminhadas, ginástica, entre outras, incluindo a adesão aos trabalhos comunitários da região, como encontros de pastoral ou os grupos de qualidade de vida, por exemplo. Uma observação semelhante foi encontrada no trabalho de Sales e Dimenstein (2009), mostrando que a atuação pautada em um modelo de atendimento predominantemente clínico e preferencialmente individual dá pouca ênfase à realidade sociocultural onde os usuários vivem. Nesse estudo também são apontadas a falta de articulação da prática com as demandas sociais e dificuldades em relação ao trabalho multiprofissional (Sales e Dimenstein, 2009).

Não se pretende negar ou anular a resposta resolutiva para muitos casos e que envolve ações altamente técnicas e tradicionais, como a psicoterapia e a consulta psiquiátrica clássica, com prescrição de medicação. Em contrapartida, as oficinas de artes, de artesanato, de relaxamento e outras práticas corporais também são necessárias e podem ser eficazes. Abib e colaboradores (2010) desenvolveram oficinas de futebol com usuários de um CAPS e verificaram que a participação no jogo podia potencializar a organização pessoal e o protagonismo, além de propiciar o envolvimento dos sujeitos nas comunidades onde vivem, favorecendo sua reinserção social.

Sabe-se que muitos dos problemas poderiam ser minimizados ou até evitados ao se olhar para eles sob a ótica da reabilitação psicossocial, entendida como “...um processo de reconstrução, um pleno exercício de cidadania e [de] [...] contratualidade no cenário das relações familiares, da rede social e do trabalho com valor social" (Saraceno, 1996, p.17 apud Costa-Rosa, 2003, p. 25). O que se depreende dessa afirmação é que, operar na lógica da reabilitação significa o envolvimento de todos os que convivem com o portador de transtorno mental, conferindo uma relação de corresponsabilidade pelo seu cuidado e projeto de vida.

Nesse sentido, a partir da presente experiência, foi possível efetivar nas UBSFs grupos de artesanato, ginástica, entre outros, incentivando também a continuidade de grupos de qualidade de vida e caminhadas. Essas atividades já eram desenvolvidas pelas equipes locais e às vezes com ajuda de voluntários, membros da comunidade.

As propostas para os casos desencadeavam ações a serem desenvolvidas, que se diversificavam: a) poderia ser uma indicação restrita e circunscrita às equipes de referência (na ESF); b) poderia envolver ambas as equipes (referência e Apoio); ou, ainda, c) poderia envolver atividades terapêuticas específicas das especialidades do Apoio (psicoterapias, grupos de terapia ocupacional, avaliação psiquiátrica, atendimento a familiares, grupos de projeto de vida, entre outras).

O critério que estabelecia o local e o tipo de ações a serem desenvolvidas era definido pelo grau de comprometimento dos sujeitos envolvidos em função de suas patologias, pela complexidade dos casos e pelos recursos profissionais disponíveis nas equipes. Nesse modo de organização, foi pactuada a corresponsabilidade dos serviços pelos casos, evitando-se seu mero encaminhamento. Assim, o serviço de origem tinha sempre informação e participação durante todo o processo de atendimento do caso na unidade de apoio e suporte quando do retorno do caso para seguimento na UBSF.

$\mathrm{O}$ atendimento a crianças e jovens também não diferiu do modo de organização e integração de serviços proposto. A fim de respeitar o princípio da integralidade da atenção, verificou-se a necessidade de incluir os pais ou responsáveis nas ações (atendimento à família) e de utilizar a rede de recursos da comunidade para intervenções voltadas a aspectos educacionais, sociais, culturais e outros.

A experiência em tela evidenciou uma possibilidade de construção coletiva do trabalho em saúde mental, por meio da aplicação, revisão e transformação contínua e compartilhada de soluções para as questões levantadas pelas equipes. Isso equivale a dizer que, em se tratando de questões complexas e de uma proposta de trabalho longitudinal (Campos e Domitti, 2007), não há soluções prontas e/ou passíveis de repetição pura e simplesmente. Muitas vezes o mesmo caso demanda condutas diversas, retornos, retomadas de discussão, ou outro procedimento ao longo do atendimento.

Assim, a estratégia do apoio sustentou-se na discussão de casos, na capacitação e fortalecimento da 
autonomia dos profissionais e em práticas muitas vezes inusitadas, criadas a partir dos recursos disponíveis. Como bem aponta Barros e colaboradores (2007, p. 816), a “... invenção de novas formas de produzir saúde passa obrigatoriamente pela modificação do processo de trabalho das equipes implicando a apropriação coletiva do desafio da construção de novas formas de responsabilização...”. Este aspecto envolve a dimensão cuidadora das práticas de saúde por meio das quais se propõe a transformação dos serviços. Há também a dimensão tecnológica envolvida na ação de saúde. Ambas as dimensões são necessárias para o acolhimento, enfrentamento e resolução do sofrimento mental. Considera-se que as práticas inovadoras são aquelas que levam em conta as necessidades do sujeito e a oferta dos recursos de saúde, mas sem se descuidar da aplicação das tecnologias desenvolvidas visando o cuidado e a cura. Portanto, ao enfrentar a complexidade dos transtornos mentais, tanto o saber técnico-científico como o senso comum devem ser utilizados, especialmente em se tratando da Atenção Básica.

A presente experiência mostrou que esse tipo de ação contribui para a efetivação da Atenção em Saúde Mental e dos objetivos da ESF nos aspectos mais gerais da assistência e no campo específico do sofrimento psíquico, promovendo a inclusão social. A função dos profissionais e a dinâmica de funcionamento das equipes (referência e apoio) tiveram um papel fundamental para alcançar os objetivos propostos pela implementação desta forma de atendimento.

No que diz respeito aos aspectos preventivos e promocionais à saúde, a ESF possui uma estrutura facilitadora para a identificação de fatores de risco existentes em determinado território. Portanto, é esperado que essas equipes possam atuar, em seu nível de competência, para minimização desses fatores.

Por outro lado, sabe-se que apesar dos avanços da psiquiatria moderna e da divulgação das psicoterapias entre a população, ainda há que se caminhar no sentido de incluir no consenso da população e dos próprios trabalhadores de saúde o fato de que algumas práticas adotadas como estilo de vida possam ser potencializadoras do bem-estar físico e mental dos indivíduos. A indicação e o incentivo dessas práticas que podem ser consideradas saudáveis pos- suem valor relativo em cada caso, mas, no entanto, podem ser eficazes na maioria das situações. Se a prática da "caminhada", por exemplo, ou de algum esporte, ou a participação em "grupos de qualidade de vida", têm alto poder na promoção e na prevenção dos agravos mentais ou emocionais leves, também podem ser de grande valia na recuperação e no tratamento complementar dos transtornos mentais graves, evitando a cronificação e facilitando a inclusão. No entanto, o momento ainda é de transição entre um modo de tratar o portador de transtorno mental medicalizando exclusivamente e um modo psicossocial, que contempla a saúde como decorrente de múltiplos fatores, não apenas o biológico.

A apropriação dos problemas de saúde mental pelas equipes de Saúde da Família vai ao encontro do conceito de integralidade (Mattos e Pinheiro, 2003) e da reforma psiquiátrica brasileira, de modo a não estigmatizar o usuário e reconhecendo-lhe um lugar na sociedade. Por outro lado, quanto mais os problemas forem identificados e tratados precocemente, menores os efeitos de cronificação e de institucionalização. Consequentemente há uma melhor qualidade de vida do indivíduo acometido pelo transtorno mental.

Uma consideração importante e que condiz com as diretrizes do SUS, é que ao se tratar na Atenção Básica o maior número de agravos, facilita-se o acesso aos serviços especializados daqueles que de fato dele necessitam. 0 atendimento resolutivo dos casos por meio da assistência aos portadores de problemas de menor complexidade ou com quadros estabilizados pode ficar sob responsabilidade da Atenção Básica, desde que devidamente apoiada técnica e institucionalmente. Esse aspecto justifica a necessidade do trabalho realizado por uma equipe de apoio, na função de sensibilizar e capacitar as equipes de ESF, favorecendo a hierarquização e integração das ações, uma vez que os serviços secundários podem se ocupar dos casos que de fato necessitem cuidados desse nível de complexidade.

De acordo com o registro de atendimentos realizados no período investigado, verificou-se que os serviços de apoio e a ESF trabalhando de forma integrada conseguiram dar resposta aos problemas de saúde mental identificados na região sob sua responsabilidade. Entretanto, para que se possa avaliar 
a eficácia no tratamento dos casos, seria necessária a realização de outros estudos, além de se analisar de maneira mais aprofundada o aspecto de estimativa de cobertura, o que não foi o objetivo deste artigo.

Nas discussões com as equipes, a reflexão girava em torno da utilização dos recursos ao alcance da população e dos profissionais, privilegiando o trabalho intersetorial, de modo a agregar as instâncias: judiciárias, educacionais e de desenvolvimento social, entre outras. Ao adotar esta estratégia, partiu-se do pressuposto de que o trabalho deve ser realizado em conjunto, porque a natureza humana é complexa e a resolução de problemas mentais não é uma tarefa simples nem pode ser realizada isoladamente. Com isso, novas e diversas possibilidades de intervenção podem se abrir e é possível propor tratamentos voltados às singularidades e com a participação dos sujeitos envolvidos (Brasil, 2008a, p. 10).

\section{Considerações finais}

Os resultados da experiência permitiram afirmar que a implementação da metodologia de Apoio Matricial pode contribuir para a construção de práticas baseadas na lógica da reabilitação psicossocial.

Os objetivos propostos inicialmente junto às equipes de SF puderam ser alcançados. Dito de outro modo, foi possível sensibilizar os profissionais da Atenção Básica em relação aos problemas de saúde mental da população que estava sob seus cuidados; capacitá-los a identificar esses problemas e instrumentá-los para o cuidado dos problemas de saúde mental de menor complexidade.

Considera-se que, no trabalho desenvolvido, pôde-se estabelecer como horizonte o respeito aos direitos do portador de transtornos mentais, além de considerar os limites e possibilidades dos profissionais, dos serviços de saúde, e, especialmente, dos usuários e seus familiares.

A dinâmica que caracterizou os processos de trabalho dos profissionais foi determinada pelo seu modo de se colocar e de lidar com as questões envolvendo o cuidado aos portadores de transtornos mentais. Esse funcionamento teve uma importância fundamental para a realização satisfatória da tarefa que aqui foi descrita, tendo sempre como norte a implantação da política de saúde mental no SUS, favorável à humanização das práticas e condizente com a Reforma Psiquiátrica no Brasil.

Assim, a intervenção realizada mostrou que por meio do apoio matricial pode ser alcançada a necessária (re)organização dos serviços para acolher à demanda por uma saúde mental de qualidade. E, ainda, que esse modo de operar condiz com a ética da construção compartilhada do espaço intersubjetivo do cuidado em saúde.

Considera-se, ainda, que a construção do processo de trabalho foi alicerçada: a) na contínua (re) organização interna do serviço de Apoio para receber os usuários referidos; b) na operacionalização de visitas e reuniões com as equipes da ESF de forma sistemática e sustentada; e c) na diversificação e oferta das práticas legitimadas nos equipamentos (seja no Apoio, seja na ESF).

A experiência permitiu evidenciar também a importância do compartilhamento de informações e saberes entre os profissionais no cotidiano de suas ações, tornando possível uma visão integrada do atendimento. Dispositivos como reuniões periódicas e sistemáticas devem ser criados e instituídos, pois contribuem para a concretização dessa finalidade.

Ainda que a intervenção tenha se encerrado em função de mudança na gestão, ela ainda gera frutos no sentido da manutenção da lógica de acolhida aos portadores de transtornos mentais na unidade de apoio, priorizando a escuta qualificada e o vínculo, na busca da integração respeitosa com as UBSFs.

\section{Agradecimentos}

Aos profissionais da Unidade Básica de Saúde de Apoio e das Unidades Básicas com Estratégia de Saúde da Família envolvidas neste trabalho, pelas informações gentilmente oferecidas.

\section{Referências}

ABIB, L. T.; et al. Práticas corporais em cena na saúde mental: potencialidades de uma oficina de futebol em um centro de atenção psicossocial de Porto Alegre. Pensar Práticas; Goiânia, v. 13, n. 2, p. 1-15, maio/ago. 2010. 
BARROS, S.; OLIVEIRA, M. A. F. de; SILVA, A. L. A. Práticas inovadoras para o cuidado em saúde. Revista da Escola de Enfermagem USP, São Paulo, V. 41, p. 815-819, dez. 2007.

BRASIL. Lei n. 10.216, de 6 de abril de 2001. Dispõe sobre a proteção e os direitos das pessoas portadoras de transtornos mentais e redireciona o modelo assistencial em saúde mental. Brasília, DF, 6 abr. 2001. Disponível em: <http://dtr2004.saude. gov.br/susdeaz/legislacao/arquivo/12_Lei_10216. pdf >. Acesso em: 10 set. 2008.

BRASIL. Ministério da Saúde. Secretaria de Atenção à Saúde. Departamento de Ações Estratégicas. Saúde Mental no SUS: Centros de Atenção Psicossocial. Série F. Comunicação e Educação em Saúde. Brasília, DF, 2004.

BRASIL. Ministério da Saúde. Secretaria de Atenção à Saúde. DAPE. Coordenação Geral de Saúde Mental. Reforma psiquiátrica e política de saúde mental no Brasil. In: CONFERÊNCIA REGIONAL DE REFORMA DOS SERVIÇOS DE SAÚDE MENTAL: 15 ANOS DEPOIS DE CARACAS. OPAS. Brasília, DF, 2005.

BRASIL. Ministério da Saúde. Secretaria de Atenção à Saúde. Núcleo Técnico da Política Nacional de Humanização. Grupo de Trabalho de Humanização. 2. ed. Brasília: Editora do Ministério da Saúde, 20o6. (Série B. Textos Básicos de Saúde).

BRASIL. Ministério da Saúde. Secretaria de Atenção à Saúde. Núcleo Técnico da Política Nacional de Humanização. Clínica ampliada, equipe de referência e projeto terapêutico singular. 2. ed. Brasília: Editora do Ministério da Saúde, 2008a. (Série B. Textos Básicos de Saúde).

BRASIL. Ministério da Saúde. Gabinete do Ministro. Portaria $n^{\circ} 154 / 2008$. Sobre os núcleos de apoio à saúde da família (NASF). Brasília, DF, 2008b.

CAMPOS, G. W. de S. Equipes de referência e apoio especializado matricial: um ensaio sobre a reorganizaçäo do trabalho em saúde. Ciência \& Saúde Coletiva, Rio de Janeiro, v. 4, n. 2, p. 393403, 1999.
CAMPOS, G. W. de S.; AMARAL, M. A. A clínica ampliada e compartilhada, a gestão democrática e redes de atenção como referenciais teóricooperacionais para a reforma do hospital Ciência \& Saúde Coletiva, Rio de Janeiro, v. 12, n. 4, jul./ago. 2007.

CAMPOS, G.W. de S., DOMITTI, A. C. Apoio matricial e equipe de referência: uma metodologia para gestão do trabalho interdisciplinar em saúde. Cadernos de Saúde Pública, Rio de Janeiro, v. 23, n. 2, fev. 2007.

CAMPOS, R. T. O. et al. Avaliação da rede de centros de atenção psicossocial: entre a saúde coletiva e a saúde mental. Revista de Saúde Pública, São Paulo, v. 43, p. 16-22, ago. 2009. Suplemento 1.

COSTA-ROSA, A. da. 0 modo psicossocial: um paradigma das práticas substitutivas ao modo asilar. In: AMARANTE, P. (Org.). Ensaios: subjetividade, saúde mental e sociedade. Rio de Janeiro, Fiocruz, 200o. p. 141-168.

COSTA-ROSA, A. da; LUZIO, C.A.; YASUI, S. Atenção psicossocial: rumo a um novo paradigma na Saúde Mental Coletiva. In: AMARANTE, P. (Org.). Archivos de saúde mental e atenção psicossocial. Rio de Janeiro: NAU, 2003. p. 13-44.

FLICK, U. Introdução à pesquisa qualitativa. Tradução J. E. Costa. 3. ed. Porto Alegre: Artmed, 2009.

HIRDES, A. A reforma psiquiátrica no Brasil: uma (re)visão. Ciência \& Saúde Coletiva, Rio de Janeiro, v.14, n.1, p. 297-305, jan./fev. 2009.

MATTOS, R. A.; PINHEIRO, R. A. Construção da integralidade: cotidiano, saberes e práticas em saúde. Rio de Janeiro: UERJ, IMS, Abrasco, 2003.

MOYSÉS, S. J. Reformar opiniões. Divulgação Saúde em Debate, Londrina, v. 8, p. 26-30, maio, 1992.

NUNES, M. de O. et al. O agente comunitário de saúde: construção da identidade desse personagem híbrido e polifônico. Cadernos de Saúde Pública, Rio de Janeiro, v. 18, n. 6. nov./dez. 2002. 
PEIRANO, M. G. S. A favor da etnografia. Rio de Janeiro: Relume-Dumará, 1995.

SALES, A. L. L. F. DIMENSTEIN, M. Psicólogos no processo de reforma psiquiátrica: práticas em desconstrução? Psicologia em Estudo, Maringá, v. 14 , n. 2, p. 277-285, jun. 2009.

SANTOS, M. Metamorfoses do espaço habitado: fundamentos teórico e metodológico da geografia. São Paulo: Hucitec, 1988. Disponível em: <http://geolink.files.wordpress.com/20o8/o6/ metamorfose-do-espaco-habitado-milton-santos. pdf $>$. Acesso em: 10 mar. 2009.

STARFIELD, B. Atenção primária: equilíbrio entre necessidades de saúde, serviços e tecnologia.

Brasília: UNESCO/Ministério da Saúde, 2002. 